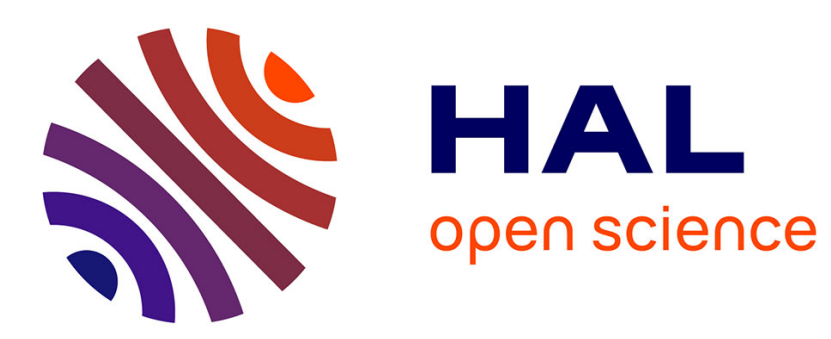

\title{
La prise en charge médicale et ostéopathique de l'énurésie nocturne primaire isolée
}

Emmanuel Burguete, Thibault Dubois

\section{To cite this version:}

Emmanuel Burguete, Thibault Dubois. La prise en charge médicale et ostéopathique de l'énurésie nocturne primaire isolée. Soins Pédiatrie/Puériculture, 2013, 34 (272), pp.17-19. 10.1016/j.spp.2013.03.002 . hal-02074615

\section{HAL Id: hal-02074615 https://hal.science/hal-02074615}

Submitted on 22 Mar 2019

HAL is a multi-disciplinary open access archive for the deposit and dissemination of scientific research documents, whether they are published or not. The documents may come from teaching and research institutions in France or abroad, or from public or private research centers.
L'archive ouverte pluridisciplinaire HAL, est destinée au dépôt et à la diffusion de documents scientifiques de niveau recherche, publiés ou non, émanant des établissements d'enseignement et de recherche français ou étrangers, des laboratoires publics ou privés. 


\title{
La prise en charge médicale et ostéopathique de l'énurésie nocturne primaire isolée
}

\author{
Auteurs : \\ Emmanuel Burguete, Ostéopathe à Saint-Médard-en-Jalles, master 1 en sciences de \\ l'éducation, DU de méthodes de recherche clinique, membre d'IRSOA (Institut de recherche \\ en sciences ostéopathiques et andragogie) et formateur au collège ostéopathique de \\ Bordeaux et Eurostéo. \\ 1 rue François Mitterrand, 33160 Saint-Médard-en-Jalles \\ emmanuel-burguete@recherche-osteopathie.org \\ Thibault Dubois, Ostéopathe à Levallois-Perret, DU de recherche clinique, Master 2 (MSc) \\ de Santé publique \\ 55 rue Chaptal 92300 Levallois-Perret
}

Mots clefs: Enurésie nocturne primaire isolée ; traitement ostéopathique ; dysfonction somatique

\section{Résumé :}

L'énurésie nocturne primaire isolée (ENPI) est un trouble fonctionnel. Son traitement conventionnel est hygiéno-diététique, rééducatif avec un système d'alarme et médicamenteux. Les résultats thérapeutiques parfois insuffisants amènent les patients à chercher des solutions complémentaires ou alternatives. L'ostéopathie est une profession qui traite les troubles fonctionnels comme l'ENPI. Cependant aucune étude n'a pour l'instant été conduite sur le sujet pour en préciser l'efficacité.

\begin{abstract}
:
The medical and osteopathic management of isolated primary nocturnal enuresis. Isolated primary nocturnal enuresis is a functional disorder. Its conventional treatment is based around lifestyle changes, rehabilitation with an alarm system and medication. Osteopathy offers a complementary or alternative treatment but studies need to be carried out to determine its effectiveness.
\end{abstract}

\section{Généralités :}

L'énurésie nocturne (EN) (1) peut faire partie de ces motifs de consultation où les spécialistes ne retrouvent fréquemment pas de causes organiques qui à elle seule puisse expliquer la symptomatologie. Ce n'est alors ni une maladie ni un état complet de bien-être physique, mental et social, signe de bonne santé selon l'OMS (2). L'EN est dite primaire lorsqu'un enfant de plus de 5 ans n'a jamais eu de période de continence pendant plus de 6 mois consécutifs. Un enfant qui est à nouveau incontinent la nuit après 6 mois minimum de 
propreté est considéré comme étant victime d'énurésie nocturne secondaire. L'énurésie nocturne primaire est dite isolée (ENPI) ou mono symptomatique s'il n'existe aucun autre symptôme concernant l'appareil urinaire en dehors de la nycturie (3). Un enfant est généralement propre la journée autour de l'âge de 3 ans à $+/-1$ an selon l'échelle de Gesell. Le contrôle et la maturité sphinctérienne vésicale se situent à l'âge de 5 ans. On considère que l'ENPI en France concerne plus de 400000 enfants de 5 à 10 ans (4) (5). Sa fréquence est de 4 à $8 \%$ des enfants âgés de 7 à 8 ans (6). Elle touche surtout les garçons avec un sex ratio est de $3 / 1$. De 0,5 à $3 \%$ (7) (8) (9) des adultes échapperaient au traitement ou à l'évolution naturellement résolutive de cette affection fréquente et invalidante.

\section{La physiopathologie et les troubles associés}

La physiologie vésico-sphinctérienne est soumise à un contrôle neurologique volontaire lié à l'éducation, l'apprentissage et à un contrôle automatique dit réflexe. Tous les auteurs s'accordent aujourd'hui sur le fait que l'ENPI est liée à 7 facteurs et symptômes que l'on retrouvera plus ou moins pour chaque enfant: La polyurie nocturne, la capacité vésicale réduite, la constipation, l'hérédité, la capacité d'éveil diminuée, l'hyperactivité avec les troubles de l'attention et les facteurs psychologiques. En fonction de son profil, l'enfant bénéficiera d'une réponse thérapeutique adaptée plus pertinente. Sans être forcément énurétique, des enfants sont obligés de se lever plusieurs fois la nuit pour aller uriner. Leur diurèse serait aussi importante la nuit que la journée du fait de l'absence d'un pic de sécrétion d'ADH (10). C'est leur capacité d'éveil liée à leur distension vésicale qui les permettra de ne pas uriner dans leur lit. Cette polyurie pourrait être majorée par une capacité vésicale réduite liée aussi plus ou moins à une vessie hyperactive mal contrôlée la nuit (11). Selon McGrath, la constipation aurait une prévalence élevée chez les patients qui ont une EN (12). Elle serait sûrement sous-évaluée par les parents puisqu'elle n'est pas recherchée systématiquement dans l'examen lors de la consultation. Des facteurs héréditaires sont très souvent retrouvés. II est important de demander aux familles d'interroger leurs différents membres afin que l'enquête soit la plus exhaustive possible. II ne faut pas oublier que l'EN est considérée comme honteuse. De nouveaux cas familiaux sont ainsi découverts. La prévalence des enfants hyperactifs et des troubles de l'attention est plus importante chez les patients énurétiques que dans la population générale (13). Les facteurs psychologiques seraient plus une conséquence de l'ENPI que la cause. Les répercussions sont importantes dans le climat familial et social et sont de plus en plus mal tolérées si les troubles persistent dans le temps.

\section{Les traitements conventionnels}

La majorité des troubles s'améliorant avec le temps, le traitement conventionnel de l'EN n'est pas systématique. II pourra être hygiéno-diététique, assisté par un système d'alarme, être médicamenteux ou un combiné des 3. II n'existe cependant pas de traitement miracle de I'EN. II faudra d'abord insister sur des règles hygiéno-diététiques simples afin d'autonomiser l'enfant au maximum. Ces mesures permettent à $20 \%$ des enfants de contrôler totalement leur symptomatologie (3). Ainsi, l'apport hydrique devra être reparti dans la journée entre le 
lever le matin et deux heures avant d'aller se coucher. On proposera et on rappellera à l'enfant d'aller aux toilettes plusieurs fois par jour dont au lever et au coucher. II pourra remplir en fonction de son âge un calendrier mictionnel sur 2 semaines renouvelables pour objectiver l'évolution des troubles. S'il porte une protection pour la nuit, il sera préférable qu'il la gère seul pour l'habillage et le déshabillage.

II existe des systèmes d'alarme sonores qui permettent aux enfants d'être réveillés dès qu'ils commencent à avoir une miction nocturne involontaire. Plus l'appareil sera utilisé, plus le réveil sera précoce au cours de la miction. En France ces systèmes ne sont pas pris en charge par la sécurité sociale (14).

La prescription médicamenteuse ne se fera jamais en première intention. Dans le cadre d'une polyurie nocturne avec un seuil d'éveil élevé, il pourra être proposé après l'âge de 6 ans un traitement par desmopressine, analogue structural de l'ADH. Le mode d'action repose sur la rétention temporaire d'eau dans l'organisme qui limite ainsi la formation d'urine. Dans le cadre d'une vessie hyperactive de l'enfant et en cas d'échec des autres traitements, un traitement par Oxybutynine qui est un antispasmodique anticholinergique est proposé en mono ou bithérapie avec la desmopressine. L'Oxybutynine permet une action de relâchement des fibres musculaires lisses de la vessie.

La desmopressine serait plus rapidement efficace que les alarmes mais aurait en fin de traitement la même efficacité. L'association d'un traitement par desmopressine et par alarme serait plus efficace qu'un traitement par alarme seule. À distance de la fin du traitement, l'alarme serait plus efficace cliniquement que la desmopressine.

D'autres traitements pourraient être efficaces mais bénéficient à ce jour d'un niveau de preuve insuffisant pour être recommandés. C'est le cas de l'hypnose, la psychothérapie, l' acupuncture, les herbes médicinales et la chiropratique (15). L'ostéopathie n'a encore à ce jour aucune étude référencée sur le sujet.

\section{Qu'est-ce que l'ostéopathie, comment se déroule une consultation. Le traitement ostéopathique de l'EN et de ses symptômes associés.}

L'usage professionnel du titre d'ostéopathe a été reconnu en France en mars 2002 (16). Plus de 20 millions de consultations ostéopathiques ont lieu chaque année pour environ 13 millions de patients. Les enfants de 0 à 18 ans représentent plus de $12 \%$ des patients consultant en ostéopathie (17). C'est une discipline de première intention, majoritairement d'exercice libéral, qui prend en charge des troubles fonctionnels en effectuant des actes de manipulations et de mobilisations non instrumentales (18). En moyenne, une séance dure 45 minutes (+/- 15 minutes). Elle comprend systématiquement 3 parties distinctes: l'interrogatoire, une phase de tests (exclusions médicales et tests spécifiques ostéopathiques) permettant la mise en évidence de dysfonctions somatiques et une phase de traitement ostéopathique. Les signes cliniques qui ont été associés à la dysfonction somatique articulaire sont classiquement décrits grâce à l'acronyme " SART " (Sensibilité/douleur à la palpation, Asymétrie des repères osseux, Restriction de la mobilité articulaire passive, modifications de la Texture des tissus mous environnants (19)). 
L'interrogatoire permet de cerner le motif et d'apprécier avec la phase de test si c'est une indication ostéopathique possible ou s'il faut réorienter le patient vers un médecin ou un autre praticien. L'ostéopathe met en lien les dysfonctions somatiques (20) rencontrées lors des tests et définit un plan de traitement afin de répondre au motif de consultation du patient. De plus en plus d'enfants viennent consulter pour des troubles fonctionnels comme l'énurésie, une hyper activité, une colopathie, des maux de tête, etc. Nous n'avons pas encore à ce jour de données précises sur le nombre de consultations concernant l'EN. Nous recevons en consultation des patients à tous les stades de traitement de l'ENPI. Après un échec de traitement conventionnel, avant l'âge de 6 ans qui ne sont pas encore traités par médicament (desmopressine) et ceux qui bénéficient du traitement conventionnel en mono ou bithérapie.

Le traitement ostéopathique n'est jamais prédéterminé par le motif de consultation. II est uniquement lié aux dysfonctions somatiques qui seront retrouvées lors des différents tests sur l'ensemble de la mobilité des tissus du corps (articulaire, musculaire, viscérale, fascias etc.). Ainsi toutes les possibilités de dysfonctions somatiques pour un motif donné sont à envisager. II n'est ni possible ni pertinent de rechercher l'efficacité d'une technique sur un motif ou bien même de standardiser une prise en charge avec un nombre de techniques prédéfinies. C'est l'efficacité d'une séance qui pourra être évaluée et non pas les techniques qui auront été utilisées.

Nous pensons en ostéopathie qu'un déficit de mobilité d'un tissu peut engendrer des répercussions locales ou à distance sur la mobilité et le bon fonctionnement d'autres tissus. Pour chaque facteurs et signes de l'EN en dehors de l'hérédité et des troubles psychologiques, nous donnerons quelques dysfonctions possibles que l'on pourrait retrouver lors d'un test ostéopathique. L'ensemble de ces corrections ne représentent pas une séance type.

La polyurie nocturne liée à la sécrétion de l'ADH pourrait sur le plan ostéopathique avoir deux niveaux de dysfonction. La première liée à des dysfonctions de mobilité d'organes comme le rein pourrait sur le plan vasculaire perturber l'imprégnation hormonale. La deuxième serait plus d'ordre crânienne avec des perturbations de tension sur l'axe hypothalamo-hypophysaire.

En cas de capacité vésicale réduite, certaines dysfonctions pourraient majorer l'impériosité et empêcher l'enfant de développer ses réflexes mictionnels inhibiteurs. La commande vésico sphinctérienne est mixte. Pour la commande volontaire, le nerf pudendal (S2-S4) peut être lié à des dysfonctions du sacrum, du muscle pyramidal et plus largement du bassin (contenant et contenu). Sur le plan involontaire, le système orthosympathique au niveau T10-L2 peut être perturbé par des tensions vertébrales, des piliers du diaphragme ce qui retentirait sur la phase de remplissage vésicale. Le système parasympatique par le biais du nerf pelvien (S2-S4) pourrait être impliqué par son activité excitatrice sur le detrusor et sur le relâchement sur l'urètre.

La constipation, la capacité d'éveil diminuée ou l'hyperactivité pourraient être la conséquence de dysfonctions entraînant une dysrégulation entre l'orthosympathique et le parasympathique. Le symptôme apparaîtrait avec un effet de seuil. En fonction des dysfonctions et de leur intensité, l'enfant pourrait évoluer dans un terrain plus 
orthosympathique ou au contraire parasympathique. Des dysfonctions vertébrales peuvent par exemple stimuler l'orthosympathique avec les nerfs splanchniques de T5 à T12.

\section{Conclusion}

L'EN n'est pas une maladie. C'est un trouble fonctionnel qui est résolutif avec le temps jusqu'à l'âge adulte dans $97 \%$ des cas. L'accompagnement thérapeutique, les règles hygiéno-diététiques et les traitements actuels (alarmes, médicaments) permettent de diminuer plus rapidement les symptômes. Cependant, certains patients qui ne répondent pas ou partiellement au traitement conventionnel cherchent d'autres solutions thérapeutiques. L'ostéopathie qui traite les troubles uniquement fonctionnels serait un traitement possible de I'EN. Elle n'a cependant à ce jour aucune preuve validée de son efficacité. Une absence de preuve ne signifiant pas une absence d'effet, il sera intéressant de définir quel(s) aspect(s) de l'EN l'ostéopathie peut-elle améliorer.

1. Haab F, Amarenco G, Coloby P, Grise P, Jacquetin B, Labat JJ, et al. [Terminology of lower urinary tract dysfunction: French adaptation of the terminology of the International Continence Society]. Prog Urol. 2004 Dec;14(6):1103-11.

2. Préambule adopté par la Conférence internationale sur la Santé', New York’, 19-22 juin 1946”; signé le 22 juillet 1946 par les représentants de 61 États 1946”; (Actes officiels de l'Organisation mondiale de la Santé', no. 2', p. 100) et entré en vigueur le 7 avril 1948 à la Constitution de l'Organisation mondiale de la Santé (OMS).

3. D. Aubert ÉB, J.-P. Blanc, G. Lenoir, F. Liard, H. Lottmann. Énurésie nocturne primaire isolée : diagnostic et prise en charge. Recommandations par consensus formalisé d'experts. Progrès en Urologie. 2010;20(5):343-9.

4. Cochat P. Énurésie et troubles mictionnels de l'enfant. Elsevier ed1997.

5. Collet JP, Simore MF, Cochat P. [Prevalence of nocturnal enuresis in school-age children]. Pediatrie. 1993;48(10):701-4.

6. Fergusson DM, Horwood LJ, Shannon FT. Factors related to the age of attainment of nocturnal bladder control: an 8-year longitudinal study. Pediatrics. 1986 Nov;78(5):884-90.

7. Lee SD, Sohn DW, Lee JZ, Park NC, Chung MK. An epidemiological study of enuresis in Korean children. BJU Int. 2000 May;85(7):869-73.

8. Torrens MJ, Collins CD. The urodynamic assessment of adult enuresis. $\mathrm{Br} \mathrm{J}$ Urol. 1975 Aug;47(4):433-40.

9. Hjalmas K, Arnold T, Bower W, Caione P, Chiozza LM, von Gontard A, et al. Nocturnal enuresis: an international evidence based management strategy. J Urol. 2004 Jun;171(6 Pt 2):2545-61.

10. Vande Walle $J$, Hoebeke $P$, Raes A. [Differences in the profile of nycthemeral diuresis]. Arch Pediatr. 1997;4 Suppl 1:7s-9s. 
11. Naseri $M$, Hiradfar $M$. Abnormal urodynamic findings in children with nocturnal enuresis. Indian Pediatr. 2012 May;49(5):401-3.

12. McGrath $\mathrm{KH}$, Caldwell PH, Jones MP. The frequency of constipation in children with nocturnal enuresis: a comparison with parental reporting. J Paediatr Child Health. 2008 Jan;44(1-2):19-27.

13. Okur M, Ruzgar H, Erbey F, Kaya A. The evaluation of children with monosymptomatic nocturnal enuresis for attention deficit and hyperactivity disorder. Int $\mathrm{J}$ Psychiatry Clin Pract. 2012 Sep;16(3):229-32.

14. ANAES. Evaluation des systèmes d'alarme dans le traitement de l'énurésie nocturne primaire monosymptomatique. 2003.

15. Huang T, Shu X, Huang YS, Cheuk DK. Complementary and miscellaneous interventions for nocturnal enuresis in children. Cochrane Database Syst Rev. 2011(12):CD005230.

16. Loi $n^{\circ}$ 2002-303 du 4 mars 2002 relative aux droits des malades et à la qualité du système de santé, (2002).

17. T. Dubois, J. Berthiller, J. Nourry, G. Landurier, F. Briere, S. Chardigny, et al. Douleurs en cabinet d'ostéopathie : étude prospective descriptive des motifs de consultations des patients consultant en cabinet d'ostéopathie. Douleur. 2012; Volume 13:Pages A59-A60.

18. Décret $n^{\circ} 2007-435$ du 25 mars 2007 relatif aux actes et aux conditions d'exercice de l'ostéopathie (2007).

19. AACOM Educational Council on Osteopathic Principles', Glossary on osteopathic terminology . In: Chila A editors.Foundations of Osteopathic Medicine. third ed.. Philadelphia`, PA: Lippincott Williams \& Wilkins`; 2010`;p. 1087-1110

20. Zegarra-Parodi $\mathrm{R}$ ea. Traitement ostéopathique de patients souffrant de lombalgies chroniques communes. Douleurs : Evaluation - Diagnostic - Traitement. 2012 Fevrier 2012;13(1):17-24. 